\title{
Performance Analysis of FAF Relaying M2M Cooperative Networks over $N$-Nakagami Fading Channels
}

\author{
Lingwei Xu ${ }^{1}$, Hao Zhang ${ }^{1,2}$, Xing Liu ${ }^{1}$ and T. Aaron Gulliver ${ }^{2}$ \\ ${ }^{1}$ College of Information Science and Engineering, Ocean University of China, \\ Qingdao 266100, China \\ ${ }^{2}$ Department of Electrical and Computer Engineering, University of Victoria, \\ Victoria, BCV8W 2Y2, Canada \\ gaomilaojia2009@163.com; zhanghao@ouc.edu.cn; liuxing3901@163.com; \\ agullive@ece.uvic.ca
}

\begin{abstract}
In this paper, the performance of fixed-gain amplify-and-forward $(F A F)$ relaying is investigated. An approximation for the average symbol error probability $(S E P)$ is derived for multiple-mobile-relay-based mobile-to-mobile (M2M) cooperative networks over $N$ Nakagami fading channels. The moment generating function (MGF) method is used to obtain average SEP expressions for various modulation techniques. The performance under different conditions is evaluated through numerical simulation to verify the analysis. These results show that the number of mobile relay nodes, the fading coefficient, the number of cascaded components, the relative geometrical gain, and the power allocation parameter have a significant impact on the SEP performance.
\end{abstract}

Keywords: M2M communication, N-Nakagami fading channels, moment generating function, average symbol error probability

\section{Introduction}

Mobile-to-mobile (M2M) communications has attracted significant research interest in recent years [1]. It is employed in many wireless communication systems such as mobile ad-hoc networks and vehicle-to-vehicle communications [2]. M2M communication networks allow multiple users to exchange information, share data, interact with ideas, and cooperate on common goals across geographical and time boundaries. The higher simplicity and affordability of M2M networks are achieved through the connectivity of devices, such as smartphones, personal digital assistant system and tablets [3]. When the devices are in motion, the double-Rayleigh fading model has been shown to be applicable [4]. This model is extended to double-Nakagami fading in [5]. The moment generating function (MGF), probability density function (PDF), cumulative distribution, and moments of the $N$-Nakagami distribution have been derived in closed form using Meijer's G-function [6].

Cooperative diversity has recently been proposed as an efficient solution to many challenging physical layer problems in $\mathrm{M} 2 \mathrm{M}$ communications. The pairwise error probability (PEP) for a cooperative inter-vehicular communication (IVC) system with amplify-and-forward(AF) relaying over double-Nakagami fading channels was investigated in [7]. In [8], the MGF method was used to derive approximate symbol error probability (SEP) expressions for fixed-gain AF (FAF) relaying over double-Nakagami fading channels. Closed-form lower and upper bounds on the outage probability of a twoway relaying AF M2M system over $N$-Nakagami fading channels were derived in [9].

To the best of our knowledge, the average SEP performance of multiple-mobile-relaybased M2M cooperative networks over $N$-Nakagami fading channels has not been 
reported in the literature. The single-relay case was investigated in [7]. A multiplemobile-relay dual-hop cooperative M2M system was investigated in [8], but only the approximate SEP over double-Nakagami fading channels was derived. Motivated by these results, in the present work we analyze a multiple-mobile-relay cooperative M2M system over $N$-Nakagami fading channels. In particular, the MGF method is used to obtain the approximate SEP of a multiple-mobile-relay-based M2M cooperative network for FAF relaying over $N$-Nakagami fading channels.

The rest of the paper is organized as follows. The multiple-mobile-relay-based M2M cooperative network model is presented in Section 2. In Section 3, the approximate SEP is obtained using the MGF method for several modulation techniques. Section 4 provides Monte Carlo simulation results to verify the analysis in Section 3. Finally, some concluding remarks are given in Section 5.

\section{System Model}

We consider a multiple-mobile-node cooperation model, namely a single mobile source (MS) node, $L$ mobile relay (MR) nodes, and a single mobile destination (MD) node, as depicted in Figure 1. Each node is equipped with a single pair of transmit and receive antennas and operates in half-duplex mode.

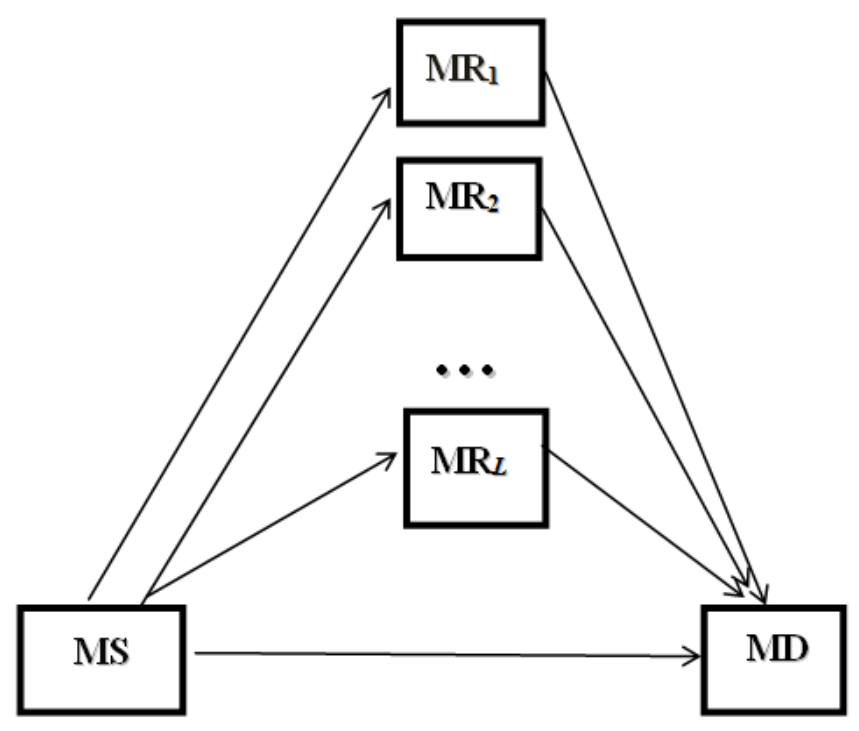

Figure 1. The System Model

Following the approach in [7], let $d_{\mathrm{SD}}, d_{\mathrm{SR} l}$, and $d_{\mathrm{RD} l}$ represent the MS to MD, MS to $\mathrm{MR}_{l}$, and $\mathrm{MR}_{l}$ to MD distances, respectively. Assuming the path loss between the MS and $\mathrm{MD}$ is unity, the relative gains of the MS to $\mathrm{MR}_{l}$ and $\mathrm{MR}_{l}$ to $\mathrm{MD}$ channels are defined as $G_{\mathrm{SR} l}=\left(d_{\mathrm{SD}} / d_{\mathrm{SR} l}\right)^{v}$ and $G_{\mathrm{RD} l}=\left(d_{\mathrm{SD}} / d_{\mathrm{RD} l}\right)^{v}$, respectively, where $v$ is the path loss coefficient [10]. We further define the relative geometrical gain $\mu_{l}=G_{\mathrm{SR} l} / G_{\mathrm{RD} l}$, which indicates the location of the $l$ th relay with respect to the source and destination [7]. When the $l$ th relay is close to the destination node, the value of $\mu_{l}$ is negative, and when it is close to the source node, the value is positive. Further, if the $l$ th relay is equidistant from the source and destination nodes, $\mu_{l}$ is $1(0 \mathrm{~dB})$.

Let $h=h_{k}, k \in\{\mathrm{SD}, \mathrm{SR} l, \mathrm{RD} l\}$, represent the complex channel coefficients of the MS to $\mathrm{MD}, \mathrm{MS}$ to $\mathrm{MR}_{l}$, and $\mathrm{MR}_{l}$ to $\mathrm{MD}$ channels, respectively, which follow an $N$-Nakagami distribution. $h$ is assumed to be the product of statistically independent, but not necessarily identically distributed, independent random variables 


$$
h=\prod_{i=1}^{N} a_{i}
$$

where $N$ is the number of cascaded components, and $a_{i}$ is a Nakagami distributed random variable with probability density function (PDF)

$$
f(a)=\frac{2 m^{m}}{\Omega^{m} \Gamma(m)} a^{2 m-1} \exp \left(-\frac{m}{\Omega} a^{2}\right)
$$

where $\Gamma(\cdot)$ is the Gamma function, $m$ is the fading coefficient and $\Omega$ is a scaling factor.

The PDF of $h$ is given by [6]

$$
f_{h}(h)=\frac{2}{h \prod_{i=1}^{N} \Gamma\left(m_{i}\right)} G_{0, N}^{N, 0}\left[\left.h^{2} \prod_{i=1}^{N} \frac{m_{i}}{\Omega_{i}}\right|_{m_{1}, \ldots, m_{N}} ^{-}\right]
$$

where $G[\cdot]$ is Meijer's $G$-function.

Let $y=\left|h_{k}\right|^{2}, \quad k \in\{\mathrm{SD}, \mathrm{SR} l, \mathrm{RD} l\}$, i.e., $y_{\mathrm{SD}}=\left|h_{\mathrm{SD}}\right|^{2}, y_{\mathrm{SR} l}=\left|h_{\mathrm{SR} l}\right|^{2}$, and $y_{\mathrm{RD} l}=\left|h_{\mathrm{RD} l}\right|^{2}$. The corresponding cumulative density functions (CDF) of $y$ can then be derived as [6]

$$
F_{y}(y)=\frac{1}{\prod_{i=1}^{N} \Gamma\left(m_{i}\right)} G_{1, N+1}^{N, 1}\left[\left.y \prod_{i=1}^{N} \frac{m_{i}}{\Omega_{i}}\right|_{m_{1}, \ldots, m_{N}, 0} ^{1}\right]
$$

By taking the first derivative of (4) with respect to $y$, the corresponding PDF is obtained as[6]

$$
f_{y}(y)=\frac{1}{y \prod_{i=1}^{N} \Gamma\left(m_{i}\right)} G_{0, N}^{N, 0}\left[\left.y \prod_{i=1}^{N} \frac{m_{i}}{\Omega_{i}}\right|_{m_{1}, \ldots, m_{N}} ^{-}\right]
$$

Based on the AF cooperation protocol, the received signals $r_{\mathrm{SD}}$ and $r_{\mathrm{SR} l}$ at the MD and $\mathrm{MR}_{l}$ nodes during the first time slot can be written as [8]

$$
\begin{gathered}
r_{S D}=\sqrt{K E} h_{S D} x+n_{S D} \\
r_{S R l}=\sqrt{G_{S R l} K E} h_{S R l} x+n_{S R l}
\end{gathered}
$$

where $x$ denotes the transmitted signal, $n_{S R l}$ and $n_{\mathrm{SD}}$ are zero mean complex Gaussian random variables with variance $N_{0} / 2$ per dimension. Here, $E$ is the total energy used by both the source and relay nodes during the two time slots. $K$ is the power allocation parameter that controls the fraction of power reserved for the broadcast phase. If $K=0.5$, equal power allocation (EPA) is used.

During the second time slot, the $l$ th relay normalizes the received signal and retransmits the resulting signal. After normalization, the received signal at the destination is given by [8]

$$
r_{R D l}=\sqrt{c_{l} E} h_{S R l} h_{R D l} x+n_{R D l}
$$

where $n_{\mathrm{RD} l}$ is a conditionally zero-mean complex Gaussian random variable with variance $N_{0} / 2$ per dimension.

For FAF relaying, $c_{l}$ is [8]

$$
c_{l}=\frac{K(1-K) G_{S R l} G_{R D l} E / N_{0}}{1+K G_{S R l} E / N_{0}+(1-K) G_{R D l}\left|h_{R D l}\right|^{2} E / N_{0}}
$$

If maximal ratio combining (MRC) is used at the MD node, the output SNR can be calculated as

$$
\gamma_{M R C}=\gamma_{S D}+\gamma_{S R D}=\gamma_{S D}+\sum_{l=1}^{L} \frac{\gamma_{S R l} \gamma_{R D l}}{1+\overline{\gamma_{S R l}}+\gamma_{R D l}}
$$

where 


$$
\begin{gathered}
\gamma_{S D}=\frac{K\left|h_{S D}\right|^{2} E}{N_{0}}=K\left|h_{S D}\right|^{2} \bar{\gamma} \\
\gamma_{S R l}=\frac{K G_{S R l}\left|h_{S R l}\right|^{2} E}{N_{0}}=K G_{S R l}\left|h_{S R l}\right|^{2} \bar{\gamma} \\
\gamma_{R D l}=\frac{(1-K) G_{R D l}\left|h_{R D l}\right|^{2} E}{N_{0}}=(1-K) G_{R D l}\left|h_{R D l}\right|^{2} \bar{\gamma} \\
\overline{\gamma_{S R l}}=K G_{S R l} E / N_{0}=K G_{S R l} \bar{\gamma}
\end{gathered}
$$

\section{Approximate Average SEP}

The MGF of $\gamma_{\text {MRC }}$ is [11]

$$
\begin{aligned}
& M_{\gamma_{M R C}}(s)=E_{x}\left[\exp \left(-s \gamma_{M R C}\right)\right] \\
& =E_{x}\left[\exp \left(-s \gamma_{S D}\right)\right] E_{x}\left[\exp \left(-s \gamma_{S R D}\right)\right] \\
& =I_{1} I_{2}
\end{aligned}
$$

where $E_{x}()$ denotes expectation.

Substituting (5) into (15), we obtain

$$
\begin{aligned}
I_{1} & =E_{x}\left[\exp \left(-s \gamma_{S D}\right)\right] \\
= & \int_{0}^{\infty} \exp \left(-s K \bar{\gamma} y_{S D}\right) f_{y}\left(y_{S D}\right) d y_{S D} \\
= & \frac{1}{\prod_{i=1}^{N} \Gamma\left(m_{i}\right)} \int_{0}^{\infty} \frac{1}{y_{S D}} \exp \left(-s \bar{\gamma} y_{S D}\right) G_{0, N}^{N, 0}\left[\left.y_{S D} \prod_{i=1}^{N} \frac{m_{i}}{\Omega_{i}}\right|_{m_{1}, \ldots, m_{N}}\right] d y_{S D} \\
= & \frac{1}{\prod_{i=1}^{N} \Gamma\left(m_{i}\right)} \int_{0}^{\infty} \frac{1}{y_{S D}} G_{0,1}^{1,0}\left[s K \bar{\gamma} y_{S D}|-\overline{0}|_{0, N}^{N, 0}\left[\left.y_{S D} \prod_{i=1}^{N} \frac{m_{i}}{\Omega_{i}}\right|_{m_{1}, \ldots, m_{N}} ^{-}\right] d y_{S D}\right. \\
= & \frac{1}{\prod_{i=1}^{N} \Gamma\left(m_{i}\right)} G_{1, N}^{N, 1}\left[\left.\frac{1}{s K \bar{\gamma}} \prod_{i=1}^{N} \frac{m_{i}}{\Omega_{i}}\right|_{m_{1}, \ldots, m_{N}} ^{1}\right]
\end{aligned}
$$

As far as we know, a suitable mathematical method to obtain $I_{2}$ exactly is still unknown. Thus, we adopt the method in [8] to obtain an approximate solution. With this approach, $\gamma_{\mathrm{RD}}$ is replaced by its expected value, so the approximate $\gamma_{\mathrm{SRD}}$ is

$$
\gamma_{S R D}{ }^{A}=\sum_{l=1}^{L} \frac{\gamma_{S R l} \gamma_{R D l}}{1+\overline{\gamma_{S R l}}+\overline{\gamma_{R D l}}}
$$

where

$$
\overline{\gamma_{R D l}}=(1-K) G_{R D l} \bar{\gamma}
$$

From Appendix A, the resulting approximation for $I_{2}$ is 


$$
\begin{aligned}
& I_{22}=E_{x}\left[\exp \left(-s \gamma_{S R D}{ }^{A}\right)\right] \\
& =\prod_{l=1}^{L} \frac{1}{\prod_{j=1}^{N} \Gamma\left(m_{j}\right) \prod_{j j=1}^{N} \Gamma\left(m_{j j}\right)} \times \\
& \int_{0}^{\infty} \frac{1}{w} \exp (-s w) G_{0,2 N}^{2 N, 0}\left[\left.\frac{w}{\bar{\gamma}} \prod_{j=1}^{N} \frac{m_{j}}{\Omega_{j}} \prod_{j j=1}^{N} \frac{m_{j j}}{\Omega_{j j}}\right|_{m_{1}, \ldots, m_{2 N}}\right] d w \\
& =\prod_{l=1}^{L} \frac{1}{\prod_{j=1}^{N} \Gamma\left(m_{j}\right) \prod_{j j=1}^{N} \Gamma\left(m_{j j}\right)} G_{2 N, 1}^{1,2 N}\left[\frac{s \overline{\gamma_{l}}}{\left.\left.\prod_{j=1}^{N} \frac{m_{j}}{\Omega_{j}} \prod_{j j=1}^{N} \frac{m_{j j}}{\Omega_{j j}}\right|_{0} ^{1-m_{1}, \ldots, 1-m_{2 N}}\right]}\right.
\end{aligned}
$$

Substituting (16) and (19) into (15), the approximate MGF of $\gamma_{\text {MRC }}$ is obtained as

$$
M_{\eta \eta}(s)=E_{x}\left[\exp \left(-s \gamma_{S D}\right)\right] E_{x}\left[\exp \left(-s \gamma_{S R D}{ }^{A}\right)\right]=I_{1} I_{22}
$$

The approximate average SEP obtained using the MGF method is then [12]

\section{1 q-ary PAM}

$$
P_{A S E P}=\sum_{d=1}^{D} E_{d} \int_{0}^{\theta_{d}} M_{r r}\left(\frac{\varphi_{d}}{V_{d}-2 \Lambda_{d} \sin ^{2} \theta}\right) d \theta
$$

For $q$-ary PAM modulation, $D=1, E_{\mathrm{d}}=2(q-1) /(\pi q), \theta_{\mathrm{d}}=\pi / 2, \varphi_{\mathrm{d}}=3 /\left(q^{2}-1\right), V_{\mathrm{d}}=0$, and $\Lambda_{\mathrm{d}=}-1 / 2$, so the average $\mathrm{SEP}$ is given by

3. 2 q-ary PSK

$$
P_{A S E P}=\frac{2(q-1)}{\pi q} \int_{0}^{\pi / 2} M_{\gamma /}\left(\frac{3}{\left(q^{2}-1\right) \sin ^{2} \theta}\right) d \theta
$$

For $q$-ary PSK modulation, $D=1, E_{\mathrm{d}}=1 / \pi, \theta_{\mathrm{d}}=(q-1) \pi / q, \varphi_{\mathrm{d}}=\sin ^{2}(\pi / q), V_{\mathrm{d}}=0$, and $\Lambda_{\mathrm{d}=}-1 / 2$, so the average SEP is given by

$$
P_{A S E P}=\frac{1}{\pi} \int_{0}^{(q-1) \pi / q} M_{\gamma \gamma}\left(\frac{\sin ^{2}(\pi / q)}{\sin ^{2} \theta}\right) d \theta
$$

\section{3. $3 q$-ary QAM}

For $q$-ary QAM modulation, $D=2$, we will consider two cases:

A. when $\theta_{\mathrm{d}}=\pi / 2, \quad E_{\mathrm{d}}=4(\sqrt{q}-1) /(\pi \sqrt{q}), \varphi_{\mathrm{d}}=3 /(2 q-2), V_{\mathrm{d}}=0, \Lambda_{\mathrm{d}=}-1 / 2$;

B. when $\theta_{\mathrm{d}}=\pi / 4, E_{\mathrm{d}}=-4(\sqrt{q}-1)^{2} /(\pi q), \varphi_{\mathrm{d}}=3 /(2 q-2), V_{\mathrm{d}}=0, \Lambda_{\mathrm{d}=}=1 / 2$;

Then the average SEP is given by

$$
\begin{aligned}
P_{A S E P} & =\frac{4(\sqrt{q}-1)}{\pi \sqrt{q}} \int_{0}^{\pi / 2} M_{\gamma \gamma}\left(\frac{3}{2(q-1) \sin ^{2} \theta}\right) d \theta \\
& -\frac{4(\sqrt{q}-1)^{2}}{\pi q} \int_{0}^{\pi / 4} M_{\gamma /}\left(\frac{3}{2(q-1) \sin ^{2} \theta}\right) d \theta
\end{aligned}
$$

\section{Numerical Results}

In this section, some numerical results are presented to illustrate and verify the average SEP results obtained in the previous section. 


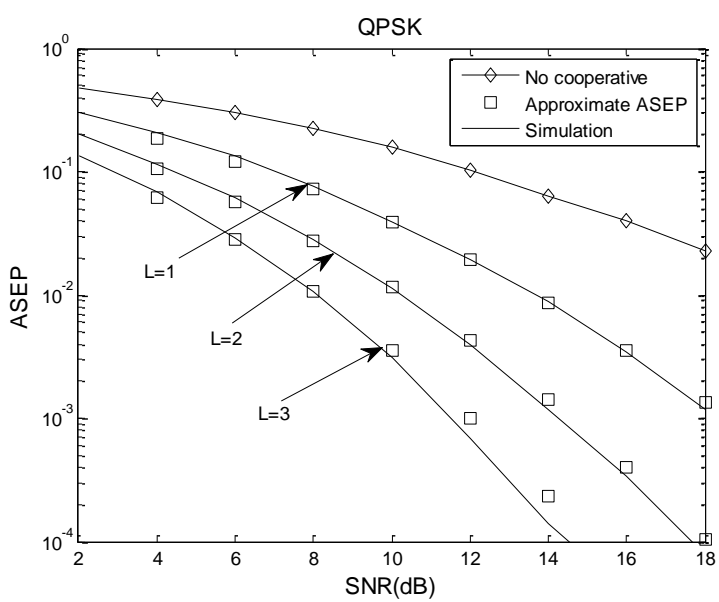

Figure 2. The Average SEP Performance over $\mathrm{N}$-Nakagami Fading Channels

Figure 2 presents the average SEP performance of a multiple-mobile-relay-based M2M cooperative network over $N$-Nakagami fading channels with QPSK modulation. The relative geometrical gain is $\mu=0 \mathrm{~dB}$, and the power allocation parameter is $K=0.5$. The number of cascaded components is $N=2$, and the number of mobile relay nodes considered is $L=1,2,3$. In all cases, the fading coefficients are $m_{\mathrm{SD}}=m_{\mathrm{SR} l}=m_{\mathrm{RD} l}=2$. This Figure shows that the numerical simulation results coincide with the theoretical results, which confirms the accuracy of the approximate average SEP. Further, the average SEP performance improves as $L$ is increased. For example, when $\mathrm{SNR}=12 \mathrm{~dB}$, the average SEP is $2 \times 10^{-2}$ with $L=1,4 \times 10^{-3}$ with $L=2$, and $1 \times 10^{-3}$ for $L=3$.

Figure 3 presents the effect of the power allocation parameter $K$ on the average SEP of a multiple-mobile-relay-based M2M cooperative network over $N$-Nakagami fading channels with various SNR values. The number of cascaded components is $N=2$, and the fading coefficient is $m=2$. The relative geometrical gain is $\mu=-10 \mathrm{~dB}$, and the number of mobile relay nodes is $L=2$. This Figure show that the average SEP performance improves as the SNR is increased. For example, when $K=0.8$, the ASEP is $7 \times 10^{-2}$ with $\mathrm{SNR}=5 \mathrm{~dB}$, $10^{-2}$ with $\mathrm{SNR}=10 \mathrm{~dB}, 7 \times 10^{-4}$ with $\mathrm{SNR}=15 \mathrm{~dB}$, and $3 \times 10^{-4}$ with $\mathrm{SNR}=20 \mathrm{~dB}$. Further, the optimum value of $K$ differs depending on the SNR. For $\mathrm{SNR}=5,10$ and $15 \mathrm{~dB}$, the optimum value of $K$ is approximately 0.8 , but for $\mathrm{SNR}=20 \mathrm{~dB}$, it is close to 0.9. This indicates that the equal power allocation (EPA) scheme is not the best scheme.

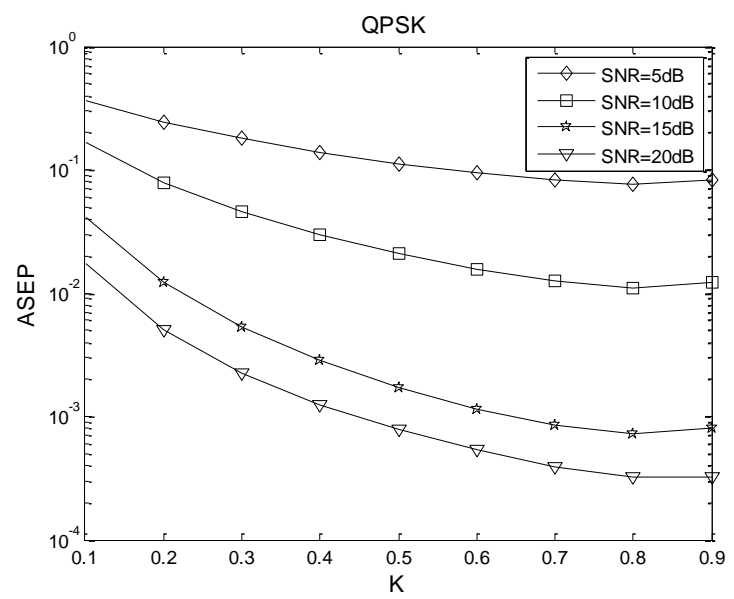

Figure 3. The Effect of the Power Allocation Parameter $K$ on the Average SEP Performance 


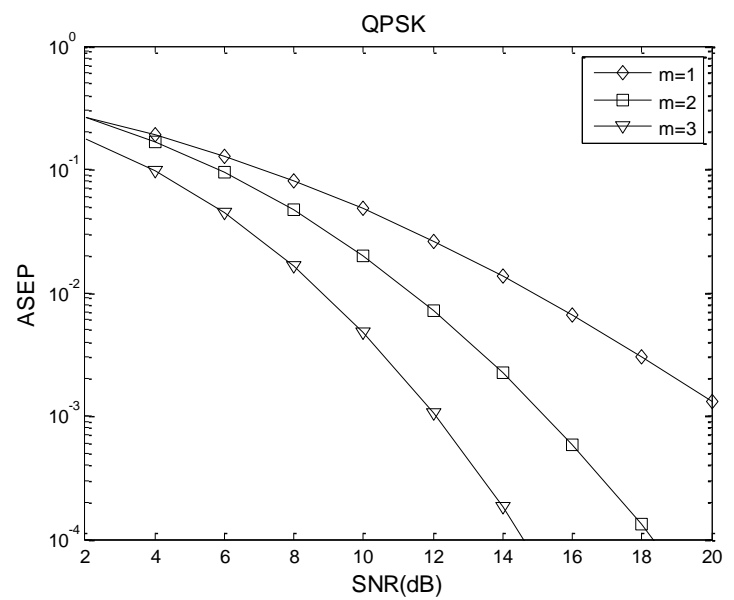

\section{Figure 4. The Effect of the Fading Coefficient $\boldsymbol{m}$ on the Average SEP Performance}

Figure 4 presents the effect of the fading coefficient $m$ on the average SEP of a multiple-mobile-relay-based M2M cooperative network over $N$-Nakagami fading channels. The number of cascaded components is $N=2$, and the fading coefficients considered are $m=1,2,3$. The relative geometrical gain is $\mu=0 \mathrm{~dB}$, and the number of mobile relay nodes is $L=3$. The power allocation parameter is $K=0.5$. This Figure shows that the average SEP improves as the fading coefficient $m$ is increased. For example, when $\mathrm{SNR}=12 \mathrm{~dB}$, the average SEP is $3 \times 10^{-2}$ with $m=1,7 \times 10^{-3}$ with $m=2$, and $1 \times 10^{-3}$ with $m=3$. This is because the fading severity of the cascaded channels weakens as $m$ is increased. For fixed $m$, an increase in the SNR reduces the average SEP, as expected.

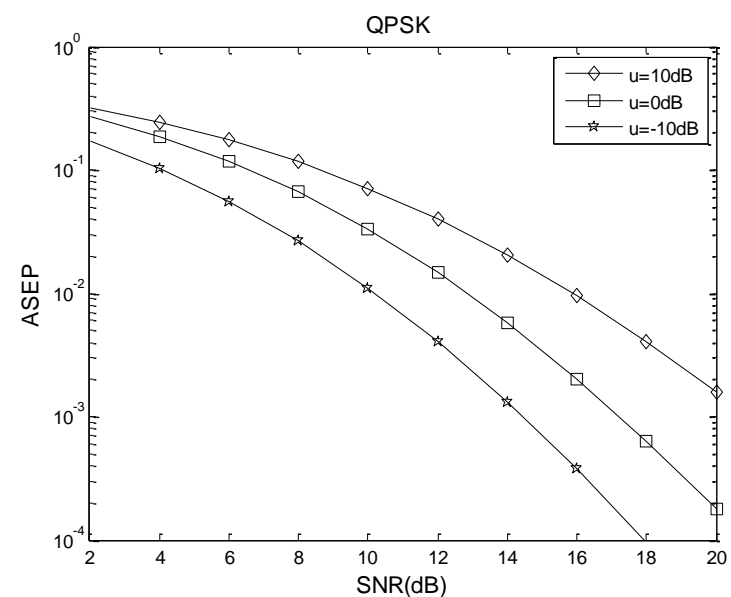

Figure 5. The Effect of the Relative Geometrical Gain $\mu$ on the Average SEP Performance

Figure 5 presents the effect of the relative geometrical gain $\mu$ on the average SEP of a multiple-mobile-relay-based M2M cooperative network over $N$-Nakagami fading channels. The number of cascaded components is $N=2$, and the fading coefficient is $m=2$. The relative geometrical gains considered are $\mu=10,0$, and $-10 \mathrm{~dB}$. The power allocation parameter is $K=0.8$, and the number of mobile relay nodes is $L=2$. This Figure shows that the average SEP improves as $\mu$ is reduced. For example, when $\mathrm{SNR}=12 \mathrm{~dB}$, the average SEP is $4 \times 10^{-2}$ with $\mu=10 \mathrm{~dB}, 1.5 \times 10^{-2}$ with $\mu=0 \mathrm{~dB}$, and $4 \times 10^{-3}$ with $\mu=-10 \mathrm{~dB}$. This 
indicates that the location of the relay should near the destination. For fixed $\mu$, an increase in the SNR reduces the average SEP, as expected.

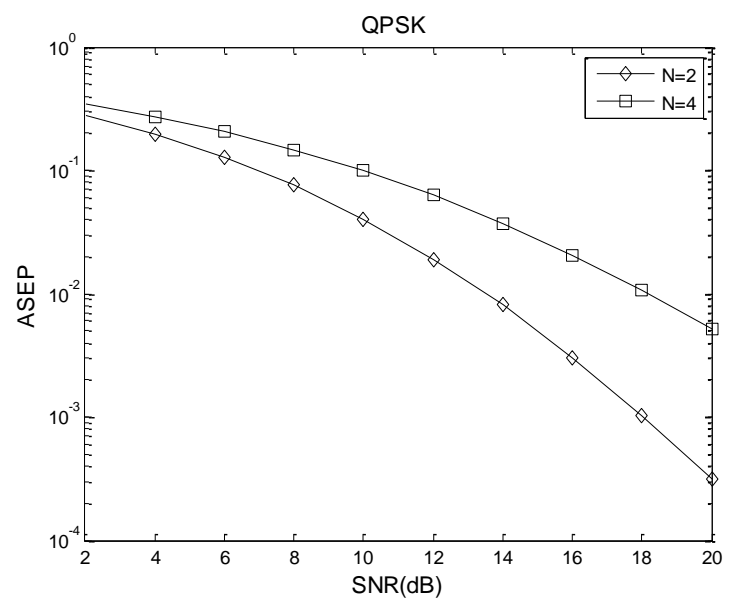

\section{Figure 6. The Effect of the Number of Cascaded Components $\mathbf{N}$ on the Average SEP Performance}

Figure 6 presents the effect of the number of cascaded components $N$ on the average SEP performance of a multiple-mobile-relay-based M2M cooperative network over $\mathrm{N}$ Nakagami fading channels. The number of cascaded components considered is $N=2,4$, which denote double-Nakagami, and 4-Nakagami fading channels, respectively. The fading coefficient is $m=2$, and the relative geometrical gain is $\mu=0 \mathrm{~dB}$. The power allocation parameter is $K=0.9$, and the number of mobile relay nodes is $L=2$. This Figure shows that the average SEP is degraded as $N$ is increased. For example, when SNR=14 $\mathrm{dB}$, the average SEP is $8 \times 10^{-3}$ with $N=2$, and $4 \times 10^{-2}$ with $N=4$. This is because the fading severity of the cascaded channels increases as $N$ is increased. For fixed $N$, an increase in the SNR reduces the average SEP, as expected.

\section{Conclusion}

In this paper, the symbol error probability (SEP) of a multiple-mobile-relay-based M2M cooperative network over $N$-Nakagami fading channels was investigated. The moment generating function (MGF) method was used to obtain approximate average SEP expressions for various modulation techniques. Analytic and simulation results were presented which show that the number of mobile relay nodes $L$, the fading coefficient $m$, the number of cascaded components $N$, the relative geometrical gain $\mu$, and the power allocation parameter $K$ can significantly influence the SEP performance. The expressions derived here are simple to compute and thus complete and accurate performance results can easily be obtained with negligible computational effort. In the future, we will consider the impact of correlated channels on the average SEP performance.

\section{Acknowledgements}

The authors would like to thank the referees and editors for providing very helpful comments and suggestions. This research was supported by the National Natural Science Foundation of China (no. 61304222 and no. 60902005), the Natural Science Foundation of Shandong Province (no. ZR2012FQ021), the Shandong Province Higher Educational Science and Technology Program (no. J12LN88), and the International Science and Technology Cooperation Program of Qingdao (no.12-1-4-137-hz). 


\section{Appendix A}

First, define two new random variables

$$
Z=\gamma_{S R l} \gamma_{R D l}, W=\frac{\gamma_{S R l} \gamma_{R D l}}{1+\overline{\gamma_{S R l}}+\overline{\gamma_{R D l}}}
$$

The PDF of $Z$ can be expressed as [13, Eq. 21]

$$
\begin{aligned}
& f_{Z}(z)=\int_{0}^{\infty} \frac{1}{x} f_{\gamma_{S R l}}(x) f_{\gamma_{R D l}}\left(\frac{z}{x}\right) d x \\
& =\int_{0}^{\infty} \frac{1}{x} \frac{1}{\prod_{j=1}^{N} \Gamma\left(m_{j}\right)} G_{0, N}^{N, 0}\left[\left.\frac{x}{\overline{\gamma_{S R l}}} \prod_{j=1}^{N} \frac{m_{j}}{\Omega_{j}}\right|_{m_{1}, \ldots, m_{N}} ^{-}\right] \times \\
& \frac{1}{z \prod_{j j=1}^{N} \Gamma\left(m_{j j}\right)} G_{0, N}^{N, 0}\left[\left.\frac{z}{x \gamma_{R D l}} \prod_{j j=1}^{N} \frac{m_{j j}}{\Omega_{j j}}\right|_{m_{1}, \ldots, m_{N}}\right] d x \\
& =\frac{1}{z \prod_{j=1}^{N} \Gamma\left(m_{j}\right) \prod_{j j=1}^{N} \Gamma\left(m_{j j}\right)} \int_{0}^{\infty} \frac{1}{x} G_{0, N}^{N, 0}\left[\frac{x}{\overline{\gamma_{S R l}}} \prod_{j=1}^{N} m_{j}||_{m_{1}, \ldots, m_{N}}\right] \times \\
& G_{0, N}^{N, 0}\left[\left.\frac{z}{x \overline{\gamma_{R D l}}} \prod_{j j=1}^{N} m_{j j}\right|_{m_{1}, \ldots, m_{N}} ^{-}\right] d x \\
& =\frac{1}{z \prod_{j=1}^{N} \Gamma\left(m_{j}\right) \prod_{j j=1}^{N} \Gamma\left(m_{j j}\right)} \times \\
& G_{0,2 N}^{2 N, 0}\left[\left.\frac{z}{\overline{\gamma_{S R l}} \gamma_{R D l}} \prod_{j=1}^{N} \frac{m_{j}}{\Omega_{j}} \prod_{j j=1}^{N} \frac{m_{j j}}{\Omega_{j j}}\right|_{m_{1}, \ldots, m_{2 N}} ^{-}\right]
\end{aligned}
$$

and the PDF of $W$ as

$$
f_{W}(w)=\frac{1}{w \prod_{j=1}^{N} \Gamma\left(m_{j}\right) \prod_{j j=1}^{N} \Gamma\left(m_{j j}\right)} G_{0,2 N}^{2 N, 0}\left[\left.\frac{w}{\gamma_{l}} \prod_{j=1}^{N} \frac{m_{j}}{\Omega_{j}} \prod_{j j=1}^{N} \frac{m_{j j}}{\Omega_{j j}}\right|_{m_{1}, \ldots, m_{2 N}}\right]
$$

where

$$
\overline{\gamma_{l}}=\frac{\overline{\gamma_{S R l}} \overline{\gamma_{R D l}}}{1+\overline{\gamma_{S R l}}+\overline{\gamma_{R D l}}}
$$

\section{References}

[1] G. Wu, S. Talwar, K. Johnsson, N. Himayat and K. D. Johnson, "M2M: From mobile to embedded internet”, IEEE Communications Magazine, vol. 49, no. 4, (2011).

[2] R. Lu, X. Li, X. Liang, X. Shen and X. Lin, "GRS: The green, reliability, and security of emerging machine to machine communications", IEEE Communications Magazine, vol. 49, no. 4, (2011).

[3] H. Munn, K. Lee, R. D. Caytiles and B. Park, "Network based Mobility Management for Smart Homes through Proxy Mobile IP", International Journal of Smart Home, vol. 8, no. 6, (2014).

[4] M. Uysal, "Diversity analysis of space-time coding in cascaded Rayleigh fading channels", IEEE Communications Letters, vol. 10, no. 3, (2006).

[5] F. K. Gong, J. Ge and N. Zhang, "SER analysis of the mobile-relay-based M2M Communication over 
double Nakagami-m fading channels", IEEE Communications Letters, vol. 15, no. 1, (2011).

[6] G. K. Karagiannidis, N. C. Sagias and P. T. Mathiopoulos, "N*Nakagami: A novel stochastic model for cascaded fading channels", IEEE Transactions on Communication, vol. 55, no. 8, (2007).

[7] H. Ilhan, M. Uysal and I. Altunbas, "Cooperative diversity for intervehicular communication: Performance analysis and optimization”, IEEE Transactions on Vehicular Technology, vol. 58, no. 7, (2009).

[8] F. K. Gong, P. Ye, Y. Wang and N. Zhang, "Cooperative mobile-to-mobile communications over double Nakagami-m fading channels", IET Communications, vol. 6, no. 18, (2012).

[9] C. Zhang, J. Ge, J. Li and Y. Hu, "Performance analysis for mobile-relay-based M2M two-way AF relaying in N*Nakagami-m fading", Electronics Letters, vol. 49, no. 5, (2013).

[10] H. Ochiai, P. Mitran and V. Tarokh. "Variable-rate two-phase collaborative communication protocols for wireless networks", IEEE Transactions on Vehicular Technology, vol. 52, no. 9, (2006).

[11] M. K. Simon and M. S. Alouini, "Digital communication over fading channels", Wiley, New York, (2004).

[12] F. Yilmaz and O. Kucur, "Exact performance of wireless multihop transmission for M-ary coherent modulations over generalized gamma fading channels", Proceedings of the International Symposium on Personal, Indoor and Mobile Radio Communications, (2008), Cannes, France.

[13] V. S. Adamchik and O. I. Marichev, "The algorithm for calculating integrals of hypergeometric type functions and its realization in REDUCE system", Proceedings of the International Symposium on Symbolic and Algebraic Computation, (2001), Tokyo, Japan.

\section{Authors}

Lingwei Xu, he was born in Shandong, China, in 1987. He received his Bachelor Degree in Department of Communication and Electronics, Qingdao Technological University, China, in 2011.From 2011 to now, he is a student of College of Information Science and Engineering, Ocean University of China. His research interests include ultra-wideband radio systems, MIMO wireless systems, and $60 \mathrm{GHz}$ wireless communications.

Hao Zhang, he was born in Jiangsu, China, in 1975. He received his Ph.D. degree in Electrical and Computer Engineering from the University of Victoria, Canada in 2004. He is now a professor at the Ocean University of China and an adjunct assistant professor at the University of Victoria. His research interests include ultra-wideband radio systems, $60 \mathrm{GHz}$ wireless communication, and MIMO wireless communication

Xing Liu, she was born in Shandong, China, in 1990.She received her Bachelor Degree from Ocean University of China, China, in 2011.From 2011 to now, and she is a student of College of Information Science and Engineering, Ocean University of China. Her research concerns UWB position.

T. Aaron Gulliver, he received the Ph.D. degree in Electrical and Computer Engineering from the University of Victoria, Victoria, BC, Canada in 1989. He is a professor in the Department of Electrical and Computer Engineering. In 2002 he became a Fellow of the Engineering Institute of Canada, and in 2012 a Fellow of the Canadian Academy of Engineering. He is also a senior member of IEEE. His research interests include information theory and communication theory, and ultra wideband communication. 